\title{
AVALIAÇÃO DA RESISTÊNCIA DE CULTIVARES E LINHAGENS DO FEIJOEIRO COMUM A DIFERENTES POPULAÇÕES DE Uromyces appendiculatus
}

\author{
GERSON P. RIOS ${ }^{1}$, EIKO M. ANDRADE ${ }^{2} \&$ JEFFERSON L. S. COSTA ${ }^{1}$
}

${ }^{1}$ Embrapa Arroz e Feijão, Caixa Postal 179, CEP 75375-000, Santo Antônio de Goiás, GO, fax: (062) 833-2100, e-mail: gerson@cnpaf.embrapa.br; ${ }^{2}$ Universidade Federal de Goiás, Cx. Postal 131, CEP 74001-970, Goiânia, GO, fax: (062) 205-1327, e-mail: eikomori@cnpaf.embrapa.br

(Aceito para publicação em 12/03/2001)

Autor para correspondência: Gerson P. Rios

RIOS, G.P., ANDRADE, E.M. \& COSTA, J.L.S. Avaliação da resistência de cultivares e linhagens do feijoeiro comum a diferentes populações de Uromyces appendiculatus. Fitopatologia Brasileira 26:128-133. 2001.

\section{RESUMO}

A técnica de folhas destacadas tem sido utilizada para estudos relacionados a doenças de plantas. Neste trabalho foi utilizada a técnica de folhas de feijoeiro (Phaseolus vulgaris) destacadas e cultivadas em vermiculita, visando estudar a resistência de diversas cultivares a diferentes populações de Uromyces appendiculatus, coletadas em diferentes regiões ou épocas. Compararam-se os sintomas da doença na planta com os observados nas folhas destacadas. Os sintomas nas folhas destacadas foram semelhantes aos das folhas nas plantas. As cultivares Novo Jalo,
Corrente e Aporé de feijoeiro foram resistentes a todas as populações do patógeno. A técnica de folhas destacadas e cultivadas em vermiculita mostrou-se útil e vantajosa com relação às demais técnicas de avaliação de resistência. Por permitir a inserção de várias folhas num mesmo recipiente, facilita a avaliação de maior número de cultivares em menor espaço, além de proporcionar melhor comodidade durante a execução do trabalho.

Palavras-chave: vermiculita, folha destacada, resistência, populações, Phaseolus vulgaris.

\section{ABSTRACT \\ Use of detached leaves to evaluate dry bean rust resistance}

The detached-leaf technique has long been used in plant disease studies. In this research, the technique was used on dry bean (Phaseolus vulgaris) leaves detached and cultivated in vermiculite to study the resistance of bean cultivars to different populations of bean rust (Uromyces appendiculatus) collected from different regions on different dates. The symptoms expressed by the whole plant were compared to those expressed on detached leaves. The lesions types in detached leaves were

\section{INTRODUÇÃO}

A ferrugem do feijoeiro (Phaseolus vulgaris L.), causada pelo fungo Uromyces appendiculatus (Pers.), está distribuída em todas as regiões onde esta leguminosa é cultivada. É considerada um dos problemas mais importantes ligados à produção do feijão (Zaumeyer \& Thomas, 1957; Ballantyne, 1974; Vargas, 1980). O patógeno é um parasita obrigatório, que completa todo o ciclo em um único hospedeiro (autóico) e produz todos os estágios de desenvolvimento (macrocíclico) (Andrus, 1931; Cummins, 1978). As pústulas desenvolvidas na superfície foliar produzem os uredosporos analogous to those on intact plants. The cultivars Aporé, Novo Jalo, and Corrente were resistant to all populations of the pathogen. The detached-leaf cultivated in vermiculite technique exhibited advantage in relation to other techniques for resistance evaluation. This technique permits the retention of more leaves in the same recipient, facilitates evaluation of more cultivars besides the economy of space and convenience in conducting the work. que infetam as plantas, dando origem a novas pústulas, ocasionando infecções sucessivas durante o ciclo vegetativo da planta. O U. appendiculatus é reconhecido como um dos patógenos de plantas com maior variabilidade patogênica. Esta variabilidade tem sido constatada através das reações de cultivares/linhagens "diferenciadoras" que, quando inoculadas com diferentes isolados do fungo, apresentam reações que variam de resistência total a alta suscetibilidade, permitindo a separação do patógeno em raças.

O cultivo de folhas destacadas tem sido utilizado há muitos anos e, já a partir de 1850, a técnica começou a ser utilizada em trabalhos ligados à fisiologia vegetal (Yarwood, 
Avaliação da resistência de cultivares e linhagens do feijoeiro comum...

1946). Em 1885 e 1917 surgiram os primeiros relatos sobre a utilização de folhas destacadas em estudos envolvendo fungos patogênicos (Farlow, 1885; Mains, 1917). Inicialmente, as folhas eram cultivadas em água, em soluções de sacarose e outras soluções com nutrientes, às vezes acrescidas de benzimidazole ou acetina (Clinton \& McCormick, 1924; Browder, 1964). A técnica empregada por Moraes \& Salgado (1982) e por Mendes \& Bergamin Filho (1986) consistiu fundamentalmente no acondicionamento de folhas, individualmente, em placas de Petri contendo uma camada de algodão e papel de filtro umedecidos em água destilada.

Com relação ao feijoeiro, Arunakumari \& Vivander (1986) conduziram ensaios de laboratório visando estudar a patogenicidade de mutantes de Xanthomonas campestris pv phaseoli Smith através da técnica de folhas destacadas. Dundas (1934, 1936, 1939, 1940a, 1940b, 1942) utilizou folhas destacadas de feijoeiro e de outras espécies para estudos de resistência ao oídio (Erysiphe polygoni DC) e à ferrugem, inclusive quanto à herança da resistência do feijoeiro a raças de $U$. appendiculatus.

Mohamed et al. (1993) enraizaram folhas de feijoeiro em vermiculita, em composto orgânico ou na mistura de ambos. Nestes substratos, contidos em vasos plásticos de 0,40 litros, foram cultivadas folhas trifolioladas obtidas de plantas com 20 dias de idade. As reações do feijoeiro a X. campestris pv. phaseoli obtidas nas folhas enraizadas foram semelhantes às das folhas não destacadas das plantas.

Rios et al. (1994a) conseguiram enraizamento em folhas destacadas de leguminosas pertencentes a 16 gêneros diferentes, além de girassol (Helianthus annuus L.), colsa (Brassica campestris L.) e nabo forrageiro (Brassica napus L.), inserindo seus pecíolos em vermiculita saturada em água destilada. Folhas de caupi [Vigna unguiculata (L.) Walp.], de feijoeiro comum e de fava (Vicia faba L.) apresentaram os melhores enraizamentos.

O cultivo de folhas de feijoeiro em vermiculita tem sido utilizado por Rios et al. (1994a, 1994b, 1995, 1996) para estudos de resistência da planta à ferrugem e a outras doenças da parte aérea do feijoeiro (Rios et al. 1994c; Rios \& Antonio, 1994; Rios et al., 1996). A técnica mostrou-se igualmente promissora no estudo da resistência do feijoeiro ao mofo branco [Sclerotinia sclerotiorum (Lib.) de Bary.] e à mela [Thanatephorus cucumeris (Frank) Donk], e em testes para avaliação da eficiência de fungicidas no controle destas doenças, bem como no controle biológico da ferrugem em feijoeiro (Rios \& Antonio, 1994; Rios et al., 1995; Trindade et al., 1996). Os resultados destas pesquisas indicaram que com esta técnica é possível obter, entre outras vantagens, maior economia sobretudo de espaço, tempo e inóculo, além da comodidade na execução dos trabalhos.

Neste trabalho objetivou-se avaliar a resistência de cultivares e linhagens de feijoeiro comum a diferentes populações de $U$. appendiculatus e investigar a viabilidade da utilização da técnica de folhas destacadas no estudo da resistência.

\section{MATERIAL E MÉTODOS}

Experimento 1. Reações de cultivares/linhagens de feijoeiro a diferentes populações de $U$. appendiculatus

\section{Obtenção e multiplicação das populações de $U$. appendiculatus}

Oito populações de $U$. appendiculatus foram obtidas nos municípios de Santo Antônio de Goiás (GO), Barreiras (BA), Monte Alegre (MG), Castro (PR), Itararé e Ribeirão Preto (SP). Cada população do patógeno consistiu de uma mistura de uredosporos obtidos de várias pústulas retiradas de folhas de feijoeiro infetadas, coletadas em diferentes pontos de áreas experimentais ou de produção. Do município de Santo Antônio de Goiás, utilizaram-se populações obtidas nos anos de 1991, 1993 e 1995. As demais populações foram coletadas durante o ano de 1995. Após serem retiradas das folhas com auxílio de pincéis, as populações foram multiplicadas na cultivar Rosinha G-2, altamente susceptível, a fim de se obter quantidade suficiente de uredosporos para a execução dos experimentos. Após as multiplicações os uredosporos foram armazenados em nitrogênio líquido $\left(-80^{\circ} \mathrm{C}\right)$ até o momento da utilização.

\section{Cultivo e inoculação das folhas destacadas}

As folhas foram obtidas em plantas aos oito dias após o plantio, desenvolvidas em casa de vegetação, em vasos com capacidade para $5 \mathrm{~kg}$, contendo três plantas/vaso. Com o auxílio de uma tesoura previamente esterilizada em álcool, as folhas primárias que haviam alcançado $2 / 3$ do desenvolvimento total foram destacadas e seus pecíolos inseridos em vermiculita saturada em água destilada na proporção de 2/1 (v/v) contida em bandejas plásticas (40x45x6 $\mathrm{cm}$ ). Para cada cultivar/linhagem (Tabelas 1 e 2) foram destacadas quatro folhas, em plantas de quatro vasos diferentes (quatro repetições). As inoculações foram efetuadas com o auxilio de um atomizador DeVilbiss, imediatamente após a inserção das folhas, utilizando-se uma suspensão contendo $2.10^{4}$ uredosporos $/ \mathrm{ml}$. Após cada inoculação, as bandejas foram envolvidas com plástico transparente e colocadas em câmara escura a $22-25{ }^{\circ} \mathrm{C}$, onde permaneceram por $48 \mathrm{~h}$. Decorrido esse período, as bandejas foram conduzidas a uma câmara de crescimento climatizada a $22-24{ }^{\circ} \mathrm{C}$ e com períodos alternados de luz/escuro de $12 \mathrm{~h}$.

As bandejas, comportando individualmente uma folha de cada cultivar, num total de 21 cultivares, foram distribuídas aleatoriamente em bancadas, num total de quatro bandejas. As avaliações foram realizadas aos 12-15 dias após as inoculações, considerando-se os tamanhos e a prevalência das lesões presentes em ambas as faces das folhas, segundo escala proposta no "1983 Bean Rust Workshop" (Stavely et al., 1983).

Experimento 2. Comparação dos sintomas nas folhas destacadas com os das folhas intactas.

Neste experimento foram utilizadas as 19 cultivares 
G. P. Rios et al.

TABELA 1 - Reações de cultivares linhagens do feijoeiro (Phaseolus vulgaris) a diferentes populações de Uromyces appendiculatus

\begin{tabular}{lccccccc}
\hline \hline \multirow{2}{*}{ Cultivar } & \multicolumn{7}{c}{ Populações de U. appendiculatus* } \\
\cline { 2 - 7 } & GO 91 & GO 93 & GO 95 & BA 95 & MA 95 & CA 95 & ITA 95 \\
\hline Novo Jalo & $2 * *$ & $2 * *$ & $1 * *$ & $2 * *$ & $3 * *$ & $2 * *$ & $1 * *$ \\
L 102005 & 5,3 & 3,5 & 4,5 & 3,4 & 3,4 & $3,4,5$ & $4,3,5,6$ \\
Ônix & 4,3 & 3,5 & $4,3,5$ & 4,3 & 3,4 & 3,4 & $4,3,5$ \\
Roxo 90 & 5,4 & $4,5,3$ & $5,4,6$ & 4,3 & 4,3 & 3,5 & $5,6,3$ \\
São José & 5,4 & 4,5 & $4,5,6$ & 3,4 & 3 & 4,5 & 4,5 \\
IPA 9 & 5,4 & 4,3 & $5,4,6$ & 3 & 3 & $3,4,5$ & 4,5 \\
Bambuí & 5,3 & 3 & 5,4 & 3,4 & 3,4 & 5,3 & $5,6,4,3$ \\
Porto Real & $5,3,4$ & 5,3 & 2,4 & 3 & 4,3 & 5,4 & $4,5,3$ \\
BR-IPA 10 & 1 & 3 & 1 & 3 & 3 & 1 & $4,5,6,3$ \\
Safira & $5,3,4$ & 3,4 & 5,6 & 3,4 & 3 & 5,4 & $4,5,3$ \\
Diamante Negro & 4,3 & 3,4 & 4,5 & 4,3 & 3 & 3,4 & $5,4,3$ \\
Xamego & 2,3 & 3,4 & 3 & 4,3 & 3,4 & $4,3,5$ & $4,5,3$ \\
Encapa-405 Goytacazes & 3,5 & 3,4 & 3,4 & 3 & 3 & 3,5 & $2,3,4$ \\
FT - Bonito & $4,3,5$ & 4,3 & 4,5 & $3,4,5$ & 3,4 & 3,4 & 4,5 \\
Corrente & 1 & 3 & 1 & 3 & 1 & 1 & 3 \\
Carioca Mg & 5,4 & 4,3 & 5,6 & 3,4 & 4,3 & $5,4,6$ & $4,5,6$ \\
Rosinha G-2 & $5,4,3$ & 4,3 & $3,4,5$ & 3,4 & 4,3 & $3,4,5$ & $5,4,3,6$ \\
TY 3364-15 & 1 & 5,3 & $2,3,5$ & 3,4 & 3,4 & 1 & 3 \\
Ouro Negro & $5,3,4$ & 3 & $3,4,5$ & 3,4 & 3,4 & 3,4 & $4,3,5$ \\
Aporéy & 2,3 & 3 & 2 & 3 & 3 & 1 & 2,3 \\
Jalo Precoce & 3,4 & 4,3 & 4,5 & 4,3 & 2,3 & 4,3 & 4,3 \\
\hline
\end{tabular}

* Procedência das populações: GO = Goiás; BA = Barreiras (Bahia); MA = Monte Alegre (MG); CA = Castro (PR); ITA = Itararé (SP); RP = Ribeirão Preto (SP).

** Reações: 1 = imune; 2 = pontos necróticos não esporulantes com diâmetros menores que $0,3 \mathrm{~mm} ; 2+=$ pontos necróticos com 0,3 - $1 \mathrm{~mm}$ de diâmetro; $2++=$ pontos necróticos com $1-3 \mathrm{~mm}$ de diâmetro; 3 = pústulas com diâmetros menores que $0,3 \mathrm{~mm} ; 4$ = pústulas com $0,3-0,5 \mathrm{~mm}$ de diâmetro; $5=$ pústulas com $0,5-0,8 \mathrm{~mm}$ de diâmetro; $6=$ pústulas com diâmetros maiores que $0,8 \mathrm{~mm}$. Onde aparece mais de um valor, eles foram ordenados de acordo com a prevalecencia, de maior para a de menor.

relacionadas na Tabela 3, diferenciadoras de raças fisiológicas de $U$. appendiculatus. Dois isolados monopustulares obtidos em plantas de feijoeiro (isolados 1 e 3 ) e dois isolados obtidos em plantas de Phaseolus acutifolius A. Gray (isolados 8 e 15), nos campos experimentais da Embrapa Arroz e Feijão, foram empregados nas inoculações. As plantas foram cultivadas em vasos de $2 \mathrm{~kg}$ em casa de vegetação. Folhas primárias destas plantas foram destacadas e seus pecíolos inseridos em vermiculita e inoculadas com suspensão do patógeno. Da mesma maneira que no experimento 1 , foram destacadas quatro folhas, em plantas de quatro vasos diferentes (quatro repetições). As técnicas de inoculação e de avaliação das folhas destacadas e intactas (seis plantas em dois vasos), foram as mesmas utilizadas no experimento 1 .

\section{RESULTADOS E DISCUSSÃO}

\section{Reações de cultivares/linhagens de feijoeiro a diferentes populações de $\boldsymbol{U}$. appendiculatus}

Nas Tabelas 1 e 2 estão apresentadas as reações das cultivares e linhagens resultantes dos Programas Nacionais e Internacionais de Melhoramento do Feijoeiro às populações de $U$. appendiculatus. Os resultados (Tabela 1) representam os tipos de lesões prevalecentes nas folhas inoculadas, de cada cultivar, de acordo com o tamanho. Considerando como reação de resistência aquelas que apresentam notas de um a três (lesões com diâmetros menores que $0,3 \mathrm{~mm}$ ), apenas as cultivares Novo Jalo, Corrente e Aporé foram resistentes a todas as populações do patógeno testadas. A cultivar BR-IPA 10 mostrou-se resistente a todas as populações do patógeno com exceção da população obtida em Itararé, no Estado de São Paulo. Foram consideradas como moderadamente resistentes as cultivares Onix, Porto Real, Xamego, Diamante Negro, Encapa 405-Goytacazes, TY 3364-15, Ouro Negro e Jalo Precoce. As populações do patógeno oriundas das regiões de Itararé (SP), Castro (PR) e Goiânia ( GO) deram origem a lesões relativamente maiores que as demais. As análises estatísticas realizadas, permitiram constatar que as correlações entre o tamanho das lesões (Tabela 1) e a percentagem da área foliar infetada (Tabela 2), foram positivas ao nível de $1 \%$ de probabilidade, para as populações GO $91\left(\mathrm{r}^{2}=0,901\right)$, GO $95\left(r^{2}=0,823\right)$, BA $95\left(r^{2}=0,720\right)$, MA $95\left(r^{2}=0,799\right)$, CA $95\left(\mathrm{r}^{2}=0,816\right)$, ITA $95\left(\mathrm{r}^{2}=0,808\right)$, RP $95\left(\mathrm{r}^{2}=0,890\right)$ e de $5 \%$ para a população GO $939\left(r^{2}=0,467\right)$. Foram também positivas, ao nível de $1 \%$, as correlações entre as reações em folhas destacadas e intactas, referentes aos isolados 1 
Avaliação da resistência de cultivares e linhagens do feijoeiro comum...

TABELA 2 - Resistência de cultivares/linhagens de feijoeiro (Phaseolus vulgaris) a diferentes populações de Uromyces appendiculatus, em folhas destacadas

\begin{tabular}{|c|c|c|c|c|c|c|c|c|}
\hline \multirow{2}{*}{ Cultivar } & \multicolumn{8}{|c|}{ Populações de $U$. appendiculatus ${ }^{*}$} \\
\hline & GO 91 & GO 93 & GO 95 & BA 95 & MA 95 & CA 95 & ITA 95 & RP 95 \\
\hline Novo Jalo & $2^{* *}$ & $2^{* *}$ & $1^{* *}$ & $2 * *$ & $3^{* *}$ & $2^{* *}$ & $1^{* *}$ & $1^{* *}$ \\
\hline L 102005 & 8 & 9 & 9 & 7 & 5 & 8 & 7 & 7 \\
\hline Ônix & 7 & 9 & 8 & 9 & 7 & 6 & 6 & 4 \\
\hline Roxo 90 & 9 & 9 & 9 & 9 & 7 & 8 & 6 & 7 \\
\hline São José & 7 & 8 & 9 & 6 & 4 & 8 & 5 & 6 \\
\hline IPA 9 & 7 & 5 & 9 & 4 & 4 & 6 & 4 & 6 \\
\hline Bambuí & 7 & 3 & 9 & 5 & 5 & 9 & 7 & 1 \\
\hline Porto Real & 5 & 7 & 8 & 3 & 6 & 7 & 7 & 8 \\
\hline BR-IPA 10 & 1 & 6 & 1 & 4 & 5 & 1 & 6 & 1 \\
\hline Safira & 8 & 7 & 9 & 4 & 5 & 8 & 4 & 1 \\
\hline Diamante Negro & 5 & 6 & 9 & 8 & 5 & 6 & 6 & 1 \\
\hline Xamego & 5 & 8 & 3 & 9 & 5 & 7 & 6 & 6 \\
\hline Encapa-405 Goytacazes & 7 & 7 & 5 & 4 & 3 & 8 & 4 & 7 \\
\hline FT - Bonito & 7 & 8 & 9 & 9 & 5 & 4 & 6 & 8 \\
\hline Corrente & 1 & 3 & 1 & 3 & 1 & 1 & 3 & 1 \\
\hline Carioca Mg & 9 & 4 & 8 & 4 & 6 & 7 & 7 & 1 \\
\hline Rosinha G-2 & 8 & 9 & 9 & 7 & 7 & 7 & 7 & 9 \\
\hline TY 3364-15 & 1 & 9 & 3 & 7 & 4 & 1 & 3 & 7 \\
\hline Ouro Negro & 8 & 5 & 8 & 7 & 5 & 6 & 5 & 1 \\
\hline Aporé & 3 & 3 & 2 & 4 & 3 & 1 & 3 & 1 \\
\hline Jalo Precoce & 5 & 7 & 5 & 7 & 3 & 4 & 7 & 5 \\
\hline
\end{tabular}

$\left(r^{2}=1,000\right), 3\left(r^{2}=0,984\right), 8\left(r^{2}=0,982\right)$ e $15\left(r^{2}=0,944\right)$.

O método de folhas destacadas de feijoeiro para avaliar a resistência a doenças já foi utilizado anteriormente, e com sucesso, para $X$. campestris pv. phaseoli (Aruna Kumari \& Vivander, 1986; Mohamed et al., 1993), E. polygoni (Dundas, 1964) e Sclerotinia sclerotiorum (Lib.) de Bary (Rios \& Antonio, 1994). O agente causal da ferrugem, U. appendiculatus, foi igualmente objeto de estudos preliminares com folhas destacadas para seleção de resistência à doença (Dundas, 1940). O sucesso desse estudo preliminar permitiu caracterizar a herança de resistência do feijoeiro à algumas raças prevalecentes na época.

\section{Comparação dos sintomas nas folhas destacadas com os das folhas intactas}

As reações das variedades de feijoeiro a ferrugem em folhas destacadas foram semelhantes às observadas nas folhas intactas (Tabela 3). Em alguns casos, a ordem na freqüência dos tipos de lesão na folha intacta foi diferente da encontrada na folha destacada, sem, contudo, alterar as reações de resistência ou de susceptibilidade das cultivares. Quando se comparam os isolados obtidos em P. acutifolius com aqueles isolados de $P$. vulgaris, observa-se maior diferenciação entre os isolados oriundos de hospedeiros pertencentes a diferentes espécies que entre isolados obtidos de uma mesma espécie. Os isolados 1 e 3, obtidos em feijoeiro, foram menos patogênicos, determinando reações de susceptibilidade (pústulas maiores que $0,3 \mathrm{~mm}$ de diâmetro) em nove das 19 cultivares, enquanto os isolados 8 e 15 , obtidos em $P$. acutifolius, foram mais patogênicos, determinando reações de susceptibilidade em 10 (isolado 8) e 12 (isolado 15), das 19 diferenciadoras. Por outro lado, as diferenciadoras México 235, Equador 299 e México 309 foram resistentes aos isolados 1 e 3 obtidos no feijoeiro e susceptíveis ao isolado 8 obtido em P. acutifolius. As cultivares Brown Beauty, CNC (Composto Negro Chimaltenango), A X S 37 (Actopan X Sanilac Seleção 37), CSW 643 (California Small White, 643) e Redlands Pioneer foram resistentes a todos os isolados, enquanto KW 814 (Kentuky Wonder 814), Olathe, Gold Gate Wax, Pinto 650 e U S 3 (United States 3) foram susceptíveis a todos eles.

Os resultados obtidos neste trabalho permitem concluir que a técnica de folhas de feijoeiro destacadas e cultivadas em vermiculita contida em bandejas é de grande utilidade, e apresenta vantagens com relação a outras técnicas tradicionalmente utilizadas na avaliação de 
G. P. Rios et al.

TABELA 3 - Reações de variedades de feijoeiro (Phaselus lunatus) à ferrugem em folhas intactas (int.) e destacadas (dest.)

\begin{tabular}{|c|c|c|c|c|c|c|c|c|}
\hline \multirow{2}{*}{ Cultivar Diferenciadora } & \multicolumn{2}{|c|}{ Isolado 1} & \multicolumn{2}{|c|}{ Isolado 3} & \multicolumn{2}{|c|}{ Isolado 8} & \multicolumn{2}{|c|}{ Isolado 15} \\
\hline & int. & dest. & int. & dest. & int. & dest. & int. & dest. \\
\hline Browm Beauty & 2 & 2 & 2 & 2 & $2^{-}$ & 2 & 2,2 & 2 \\
\hline United States 3 & $4,3,5$ & 4,5 & 4,3 & 4,3 & 4,3 & 4,5 & 4,5 & $3,4,5$ \\
\hline México 235 & 1 & 1 & 2 & 2 & 4,3 & 4,3 & 2,3 & 2,3 \\
\hline KW 765 & 3,4 & 3,4 & 3,4 & 3,4 & 3,4 & 3,4 & 3,4 & 3,4 \\
\hline $\mathrm{CNC}$ & 2,3 & 2,3 & 1 & 1 & 2 & 2 & 2,3 & 2,3 \\
\hline Equador 299 & 2,3 & 2,3 & 2 & 2 & 4,3 & 4,3 & 4 & 4,3 \\
\hline Early Gallantin & 2,3 & 2,3 & 3,4 & 4,3 & 4,5 & 4,5 & 5,6 & $4,5,6$ \\
\hline AxS 37 & 2 & 2 & 3,2 & 3,2 & 2,3 & 2,3 & 2,3 & 2,3 \\
\hline Aurora & $5,6,4$ & $5,6,4$ & 5,4 & 5,4 & 2,3 & 2,3 & 4,5 & 4,3 \\
\hline México 309 & 2 & 2 & 2 & 2 & 2,3 & $2,3,4$ & $4,5,3$ & $4,3,5$ \\
\hline CSW 643 & 2 & 2 & 1 & 1 & 1 & 1 & 1 & 1 \\
\hline KW 780 & 4,5 & 4 & 2,3 & 2,3 & 2,3 & 2,3 & 4 & 3,4 \\
\hline Pinto 650 & 4,3 & 4,3 & $5,4,6$ & $5,4,6$ & 4,3 & 4,3 & 4,3 & $4,3,5$ \\
\hline Golden Gate Wax. & 5,6 & 5,6 & 4,5 & 4,5 & 4,3 & 4,3 & 4,5 & 4,5 \\
\hline Redlands Pioneer & 2 & 2 & 2 & 2 & 2,3 & 2,3 & 2 & 2 \\
\hline NEP-2 & $4,3,5$ & $4,3,5$ & 3,4 & 3,4 & 1 & 1 & 2 & 2 \\
\hline 51051 & 2,3 & 2,3 & 2,3 & 2,3 & 2,3 & 2,3 & $4,5,3$ & $3,4,5$ \\
\hline Olathe & $4,3,5$ & 4,3 & 3,4 & 3,4 & 3,4 & 3,4 & 3,4 & 3,4 \\
\hline KW 814 & 5,6 & $5,4,6$ & 5,4 & 5,4 & 4,5 & 5,4 & 4,5 & $4,5,6$ \\
\hline
\end{tabular}

* Reações: 1 = imune; 2 = pontos necróticos não esporulantes com diâmetros menores que 0,3 mm; $2^{+}=$pontos necróticos com 0,3 - 1 mm de diâmetro; $2^{++}=$pontos necróticos com 1 - $3 \mathrm{~mm}$ de diâmetro; $3=$ pústulas com diâmetros menores que $0,3 \mathrm{~mm} ; 4=$ pústulas com 0,3 - 0,5 $\mathrm{mm}$ de diâmetro; 5 = pústulas com 0,5 - 0,8 mm de diâmetro; $6=$ pústulas com diâmetros maiores que $0,8 \mathrm{~mm}$. Onde aparece mais de um valor, eles foram organizados de acordo com a prevalecência, de maior para a de menor.

resistências genéticas às doenças da parte aérea. Pode ser útil, também, na diferenciação de raças fisiológicas de $U$. appendiculatus. As cultivares Aporé, Novo Jalo e Corrente, altamente resistentes a todas populações de $U$. appendiculatus testadas, pelo fato de serem altamente produtivas, pertencerem a diferentes grupos genéticos, com diferenciados tipos e cor dos grãos, são de fundamental importância no programa de melhoramento genético, objetivando resistência à ferrugem.

\section{REFERÊNCIAS BIBLIOGRÁFICAS}

ANDRUS, C.F. The mechanism of sex in Uromyces appendiculatus and Uromyces vignae. Journal of Agricultural Research 42:559-587. 1931.

ARUNAKUMARI, A. \& VIVANDER, A.K. Identification of Xanthomonas campestris pv. phaseoli mutants with altered pathogenicity using a detached bean leaflet bioassay. Phytophatology 76:11361986 (Abstract).

BALLANTYNE, B.J. Resistance to rust (Uromyces appendiculatus) in bean (Phaseolus vulgaris). Proceedings of the Linnean Society of the New South Wales 98:107121. 1974.

BROWDER, L.E. A modified detached- leaf culture technique for study of cereal rusts. Plant Disease Reporter 48:906908. 1964.
CLINTON, G.P. \& McCORMICK, F. A. Rust infection of leaves in Petri dishes. Connecticut Bulletin 260:475-501. 1924.

CUMMINS, G.B. Rust fungi on legumes and composites in North America. Tucson. University of Arizona Press. 1978.

DUNDAS, B. A preliminary report on the inheritance to rust (Uromyces appendiculatus) in beans (Phaseolus vulgaris). Phytopathology 30:786. 1940. (Abstract).

DUNDAS, B. Further studies on the inheritance to powdery mildew of beans Hilgardia 13:551-565. 1964.

FARLOW, W.G. Notes on some species of Gymnosporangium and Chrysomyxa of the United States. Proceedings of the American Academy of Arts and Science 20:311-323. 1885 .

MAINS, E.B. The relation of some rusts to physiology of their hosts. American Journal Botany 4:179-220. 1917.

MENDES, B.M.J. \& BERGAMIN FILHO, A. Adaptação da técnica da cultura da folha destacada para a quantificação dos parâmetros epidemiológicos monociclicos da ferrugem do feijoeiro. Fitopatologia Brasileira 11:103114. 1986.

MOHAMED, F., MOHAMED, E., ARNAUD-SANTANA \& COYNE, D.P. Rooting of bean leaves and use in germplasm evaluation for common bacterial blight resistance. Euphytica 65:1261-1266. 1993.

MORAES, S.A. \& SALGADO, C.L. Utilização da técnica 
Avaliação da resistência de cultivares e linhagens do feijoeiro comum...

de folhas destacadas de amendoim (Arachis hypogea L.) para inoculações com Cercospora arachidicola Hori e Cercospora personata (Berk. \& Curt.) Ell. \& Ev. Summa Phytopathologica 8:39-55. 1982.

RIOS, G.P. \& ANTONIO, F.G. Teste de laboratório para avaliações de resistência genética e eficiência de fungicidas no controle do mofo branco do feijoeiro. Summa Phytopathologica 20:41. 1994. (Resumo).

RIOS, G.P., ANTONIO, F.G. \& RODRIGUES, F.A. Enraizamento de folhas em vermiculita para estudos de doenças foliares. Fitopatologia Brasileira 19:268. 1994a. (Resumo).

RIOS, G.P., ANTONIO, F.G. \& SOUSA, A. Avaliação da resistência de genótipos do feijoeiro à ferrugem (Uromyces appendiculatus) em folhas enraizadas. Summa Phytopathologica 20:41. 1994b. (Resumo).

RIOS, G.P., BOTELHO, S.A., SOUZA, C.A. \& WATANAKE, M.C. Utilização de folhas enraizadas em vermiculita para avaliação de resistência genética do feijoeiro à doenças da parte aérea. Fitopatologia Brasileira 19:315. 1994c. (Resumo).

RIOS, G.P., COSTA, G.R., MELO, M.L.B. \& PAULA, R.S. Folhas de feijoeiro enraizadas em vermiculita para identificação de resistência a diversas populações de Uromyces appendiculatus. Fitopatologia Brasileira
20:363. 1995. (Resumo).

RIOS, G.P., FONTES, F.A. \& RODRIGUES, A. A. Sintomas da mancha angular em folhas enraizadas do feijoeiro: Influência da idade das plantas no tamanho das lesões. In: 5, Anais, Reunião Nacional de Pesquisa de Feijão, Goiânia, GO. 1996. pp.221-223.

STAVELY, J.R., FREYTAS, G.F., STEADMAN, J.R. \& SCHWARTZ, H.F. The 1983 bean rust workshop. Annual Report of the Bean Improvement Cooperative 26: 5-6. 1983.

TRINDADE, L.C., COSTA, J.L.S., ROCHA, A.C. \& RIOS, G.P. Uso do método de folhas enraizadas do feijoeiro nos testes de eficiência de Bacillus subtilis no controle biológico da ferrugem (Uromyces appendiculatus). In: 5, Anais, Reunião Nacional de Pesquisa de Feijão, Goiânia, GO. 1996. pp.207-209.

VARGAS, E. Rust. In: Schwartz, H.F. \& Gálvez, G.E. (Ed.). Problemas de producción del frijol: enfermedades, insectos, limitaciones edáficas y climáticas de Phaseolus vulgaris. Cali. CIAT. 1980. pp.17-36.

YARWOOD, C.E. Detached leaf culture. The Botanical Review 5:1-56. 1946.

ZAUMEYER, W.J. \& THOMAS, H.R. A monographic study of bean diseases and methods of their control. Washington. USDA. 1957. 